\title{
Dynamic Diffraction and Interband Transitions in Two-Dimensional Photonic Lattices
}

\author{
Bernd Terhalle, ${ }^{1,2,3, *}$ Anton S. Desyatnikov, ${ }^{1}$ Dragomir N. Neshev, ${ }^{1}$ Wieslaw Krolikowski, ${ }^{2}$ \\ Cornelia Denz, ${ }^{3}$ and Yuri S. Kivshar ${ }^{1}$ \\ ${ }^{1}$ Nonlinear Physics Centre, Centre for Ultra-high bandwidth Devices for Optical Systems (CUDOS), Research School of Physics and \\ Engineering, The Australian National University, Canberra, ACT 0200, Australia \\ ${ }^{2}$ Laser Physics Centre, Research School of Physics and Engineering, The Australian National University, \\ Canberra, ACT 0200, Australia \\ ${ }^{3}$ Institut für Angewandte Physik and Center for Nonlinear Science (CeNoS), Westfälische Wilhelms-Universität Münster, \\ 48149 Münster, Germany
}

(Received 1 December 2010; revised manuscript received 18 January 2011; published 23 February 2011)

\begin{abstract}
We reveal a direct link between two fundamental wave phenomena in periodic media, Pendellösung oscillations and resonant coupling between spectral bands. We experimentally measure the power transfer between laser beams associated with the high-symmetry points in periodic and biased hexagonal photonic lattices. As a result, we demonstrate that Pendellösung oscillations dominate the dynamics of resonant interband transitions on a short propagation scale.
\end{abstract}

PACS numbers: 42.25.Bs, 42.82.Et

Coherent transport of waves in periodic media manifests itself in resonant phenomena such as Bragg scattering of $\mathrm{x}$ rays, electrons, and neutrons [1] in crystals, matter waves [2], and visible light [3] in optical lattices. Resonant coupling between two [1,2,4,5] or several [6] forward and Bragg-reflected waves leads to dynamic diffraction and Pendellösung effect $[1,2,4,5]$ with the wave energy oscillating between high-symmetry momentum states. In contrast, the resonant coupling between spectral bands can be induced by an external force, and it leads to Landau-Zener interband tunneling (LZT) [7,8]. These two fundamental phenomena have never been linked before, even though they are observed in essentially similar settings, as coupling between localized momentum states [8].

Periodic photonic structures, such as coupled waveguides $[3,9,10]$ and optical lattices $[10,11]$, allow direct visualization of many fundamental linear and nonlinear phenomena inherent to wave packets and quantum particles of different nature [12], such as electrons in crystals and matter waves in optical lattices [13]. Examples include Bloch oscillations [14], LZT [7,8], as well as nonlinear $[10,11,15]$, disorderdriven [16], and dynamic [17] localization. While the later use narrow [11,15] or partially incoherent [18] beams with wide spatial spectra, broad beams with localized spatial spectra are usually required to selectively access specific Bragg resonances. For instance, one-dimensional (1D) Pendellösung oscillations between forward and Braggreflected waves have been demonstrated with quasiplane waves in holographic volume gratings [4] and in microwave photonic crystals [5]. Nevertheless, resonant effects, such as Bloch oscillations [14] and LZT [7,8] can be observed with relatively narrow (and thus experimentally accessible) beams covering only several lattice sites.

In this Letter, we reveal a deep relation between two fundamental wave phenomena, Pendellösung oscillations and interband transitions, in experimental studies of resonant coupling between high-symmetry momentum states of two-dimensional (2D) photonic lattices. In pure periodic lattices these critical points form few-level oscillatory systems, with periodic Pendellösung transfer of population between levels due to interference of two or many Bloch waves; this process is similar to Rabi oscillations and energy beating in coupled waveguides. In the regime of LZT in lattices of a finite length, the observed energy transport between high-symmetry points can be explained by Pendellösung and Bloch oscillations, without significant tunneling between spectral bands.

We begin with the resonant model of interband coupling in hexagonal photonic lattices governed by the LandauZener-Majorana (LZM) system [6,19]. As an example, we consider a two-level LZM model of two resonantly coupled plane waves (high-symmetry momentum states) with complex amplitudes $c_{1,2}$ :

$$
d c_{1,2} / d z=-i b_{1,2} z c_{1,2}+i \Omega c_{2,1} .
$$

Here, $z$ is the propagation length in the crystal, the coefficients $b_{1,2}$ are proportional to the linear gradient of the refractive index, and the coupling coefficient $\Omega$ is defined by the resonant Fourier component of the lattice potential [20]. Equation (1) describes the asymptotic transfer of populations $\left|c_{2,1}\right|^{2}$ between levels due to interband tunneling, induced by the refractive index gradient. However, at short propagation distances $z \approx 0$, close to the Bragg resonance, the gradient terms $b_{1,2} z c_{1,2}$ are small and can be neglected, thus effectively reducing Eq. (1) to

$$
d c_{1,2} / d z=i \Omega c_{2,1} .
$$

This generic oscillatory system can be also obtained from the LZM model (1) with zero index gradient, $b_{1,2}=0$, and thus it describes Pendellösung oscillations along the propagation length [5] of a perfectly periodic crystal. The oscillation frequency $\Omega$ is determined by the 
lattice depth [4]. Therefore, with initial population at one level, $c_{1}(0)=1$ and $c_{2}(0)=0$, the harmonic solution to Eq. (2) can be used to derive $\Omega$, and thus the lattice depth, as a function of the power ratio at two high-symmetry points after propagation of distance $z, \Omega z=\tan ^{-1}\left|c_{2}\right| /\left|c_{1}\right|$. In fact, a similar method is used routinely with $\mathrm{x}$-ray and neutron [1] scattering to determine the structure and atomic scattering amplitude of crystals. Interestingly, the same Eq. (2) also describes two evanescently coupled waveguides [9], and it is analogous to Rabi oscillations of an externally driven two-level atom [21]. The analogy with Rabi oscillations [6] is based on the similarity between atomic levels and high-symmetry points, with coupling strength $\Omega$ proportional to the lattice amplitude, similar to the Rabi frequency $\Omega$ defined by the strength of the driving field [21].

The arguments above suggest that Pendellösung oscillations should dominate any interband coupling at small propagation distances. Therefore, the first important step in our experimental demonstration is to use pure Pendellösung oscillations in lattices without index gradient to verify the applicability of the theoretical model and measure the lattice depth. In experiments, we realize photonic LZM systems in an optically induced hexagonal lattice produced by interfering three ordinarily polarized beams from a frequency-doubled $\mathrm{Nd}: \mathrm{YVO} 4$ laser at a wavelength of $532 \mathrm{~nm}$ in a $23 \mathrm{~mm}$ long photorefractive strontium barium niobate crystal. An external dc electric field, $E_{\text {ext }}$, applied to the crystal, is used to control the lattice depth. We use stretched hexagonal lattices, see Fig. 1(a), to restore the symmetry of intersite coupling broken by anisotropic modulation of the refractive index in the photorefractive crystal [22]. The period in horizontal $x$ direction is $d=22 \mu \mathrm{m}$ and the stretching factor is $\eta=$ 2.4. Two CCD cameras are used to analyze the output beam in real as well as in Fourier space (far field). The lattice Brillouin zone (BZ) and the band-gap spectrum are shown in Figs. 1(b) and 1(c).

The two-level resonance can be excited on the border of the irreducible BZ, and we choose the $Y$-symmetry point of the lattice where the gap between the first two Bloch bands is the smallest, as seen in the plot of the propagation constant $\beta$ in Fig. 1(c). The 1D Pendellösung oscillations between the critical points $Y$ and $Y^{\prime}$ in Fig. 1(b) are excited by sending a Gaussian beam with FWHM of $160 \mu \mathrm{m}$ in the direction of the $Y$ point and recording the far-field intensity profiles, Figs. $1(\mathrm{e})-1(\mathrm{~g})$. The input at the $Y$ point is Bragg reflected and the output contains a second peak at the $Y$ ' point. The Bragg-reflected beam remains well localized in Fourier space with its width replicating input beam. We estimate the relative powers $P_{1,2}$ by numerical integration, and compare them with the plane-wave intensities $\left|c_{1,2}\right|^{2}$ given by Eq. (2). The ratio of the two powers changes with the externally applied field, $E_{\text {ext }}=$ $0-1 \mathrm{kV} / \mathrm{cm}$, because the latter determines the lattice depth and thus the oscillation frequency, $\Omega L=\tan ^{-1} \sqrt{P_{2} / P_{1}}$, as depicted in Fig. 1(d). The resonant theory [6] predicts (a)
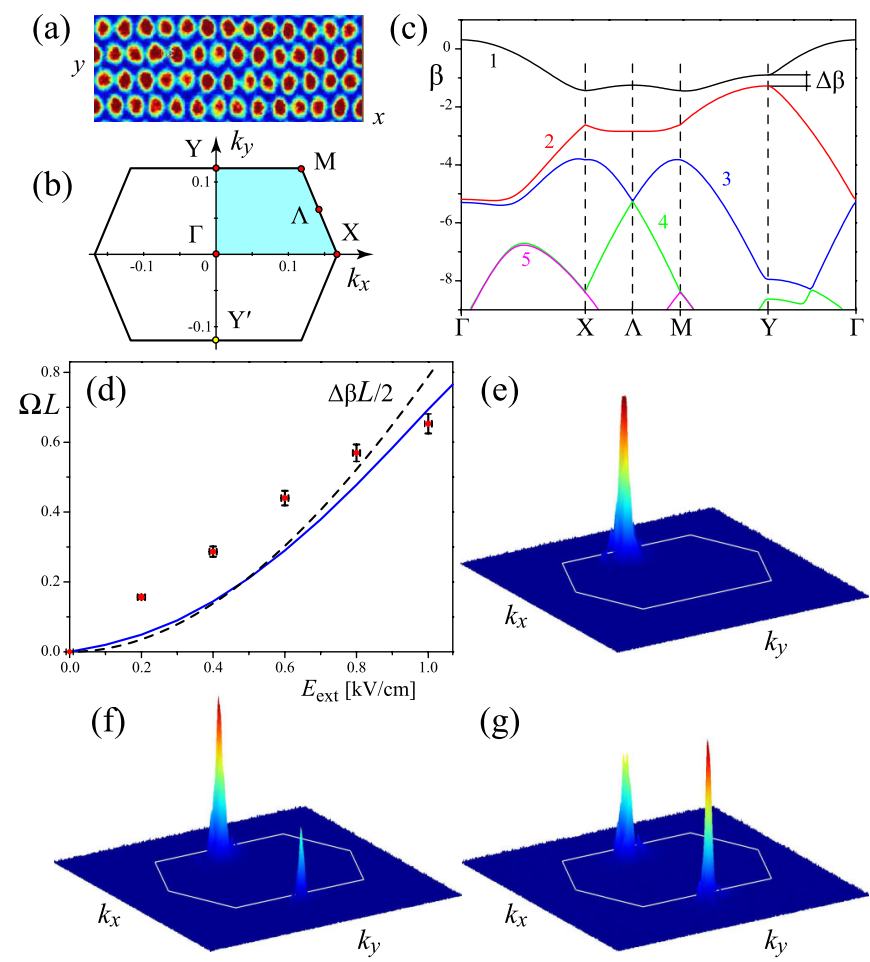

(e)

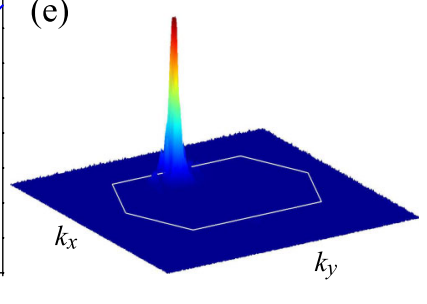

$(\mathrm{g})$

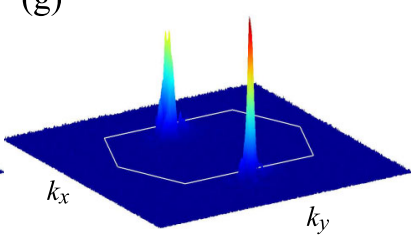

FIG. 1 (color online). (a) Experimental intensity distribution of the stretched hexagonal lattice. (b) First BZ with the highsymmetry points. (c) Band-gap diagram calculated for a latticewave amplitude $I_{0}=1.1$ and an external field $E_{\text {ext }}=1 \mathrm{kV} / \mathrm{cm}$. The numbers next to the curves show band index. (d) Experimentally measured Rabi frequency $\Omega$ of oscillations between $Y$ and $Y$ ' points (shown as dimensionless parameter $\Omega L$ ) versus external field $E_{\text {ext }}$ (dots with error bars). Solid line: numerical simulations; dashed line: normalized gap $\Delta \beta L / 2$ between first two bands at $Y$ point as indicated in (c). (e)(g) Experimentally recorded far-field output intensity for $E_{\text {ext }}=0.2,0.6$, and $1 \mathrm{kV} / \mathrm{cm}$, respectively.

Pendellösung oscillations between the two high-symmetry points due to the interference and beating of two Bloch waves. Therefore, the oscillation frequency can also be estimated as $\Omega \approx \Delta \beta / 2$. Indeed, the latter values of $\Delta \beta L / 2$ [calculated, dashed line in Fig. 1(d)] are close to the experimental data and to the results of full numerical simulations. The Bloch-wave spectrum of the input Gaussian has dominating contributions from the first and second bands (not shown) for the entire range of lattice depths, which further supports the validity of the resonant two-level approximation.

The two-level system (2) offers interesting opportunities to explore phase-only manipulation of Pendellösung oscillations in photonics. Figure 2 presents experimental results on two-beam excitation of the two-level system, with the relative phase $\delta$ between input beams at $Y$-symmetry points, providing full control over output population ratio. This input realizes the following initial conditions for the LZM system (2): $c_{1}(0)=1 / \sqrt{2}$ and $c_{2}(0)=\exp (i \delta) / \sqrt{2}$; the solution reads $\left|c_{1,2}(z)\right|^{2}=(1 \pm \sin \delta \sin 2 \Omega z) / 2$. For a given lattice depth $\Omega$ and crystal length $z=L$, the output 

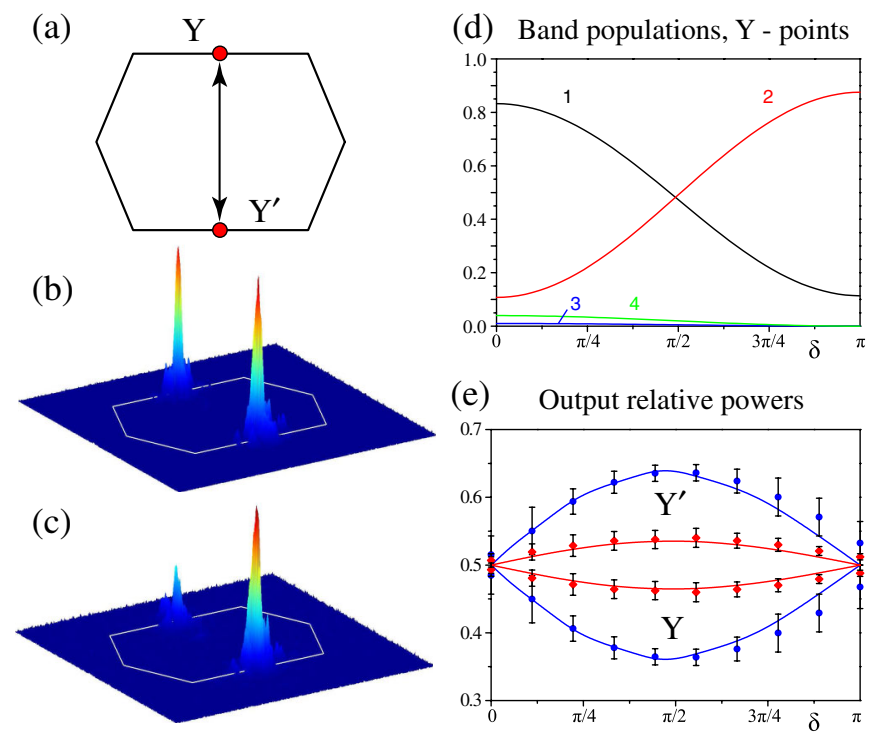

(e) Output relative powers

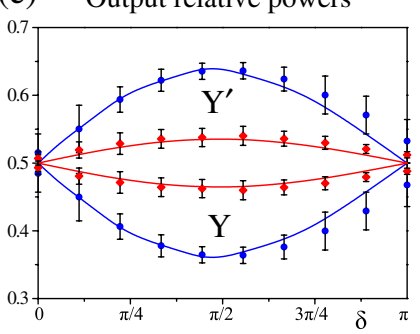

FIG. 2 (color online). Two-level Pendellösung oscillations. (a) The two-level system of Fig. 1, but excited with a pair of beams with equal power and phase difference $\delta$. (b),(c) Examples of experimental far-field output intensity distributions for (b) $\delta=0$ and (c) $\delta=\pi / 2$. (d) Corresponding Bloch-band populations, calculated for $I_{0}=1.1$ and $E_{\text {ext }}=1 \mathrm{kV} / \mathrm{cm}$. (e) The measured (relative) powers of output beams (symbols and error bars) and the corresponding numerical simulations (solid lines) for external bias $E_{\text {ext }}=0.4 \mathrm{kV} / \mathrm{cm}$ (inner curves) and $E_{\text {ext }}=1 \mathrm{kV} / \mathrm{cm}$ (outer curves). The symbols next to the curves indicate corresponding beam in BZ.

is fully defined by the input phase $\delta$. The Bloch-band population in Fig. 2(d) strongly depends on the phase difference $\delta$ and shows coupling between the two lowest Bloch bands. The experimental measurements in Fig. 2(e) show a remarkable agreement with simple harmonic solutions to Eq. (2), namely, the two cases correspond to different frequencies $\Omega$, and thus different amplitudes of power ratios at $\delta=\pi / 2$, as defined by the LZM solution: $\max \left(\left|c_{1}(L)\right| /\left|c_{2}(L)\right|\right)=(1+\sin 2 \Omega L) / \cos 2 \Omega L$.

More importantly, 2D lattices provide access to multilevel systems, in addition to the 1D Bragg reflection described above. In the configuration in Fig. 3(a), the field amplitudes at $M$ points in momentum space are described by three-level LZM model [20]. By varying the relative phase $\delta$ between two input beams at $M$ and $M^{\prime}$ ' points, we are able to distribute and switch the output power between two or three beams, in excellent agreement with resonant theory. Despite the anisotropy of the stretched hexagonal lattice, the experimental outputs in Figs. 3(c)-3(f) clearly show the strong localization at high-symmetry points. Indeed, the measured relative power of the output peaks is in excellent agreement with numerical simulations in Fig. 3(h), and it recovers the corresponding solutions to the Rabi system derived in [20].

In contrast to the Rabi oscillations between waveguide modes [23] or spectral bands [24] in longitudinally modulated waveguides, so far we observed oscillations (a)

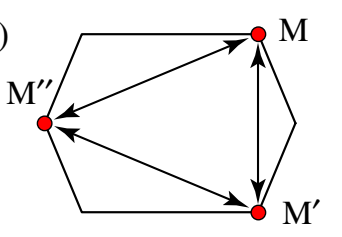

(b)

(c)

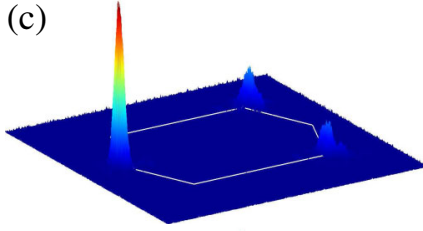

(e)

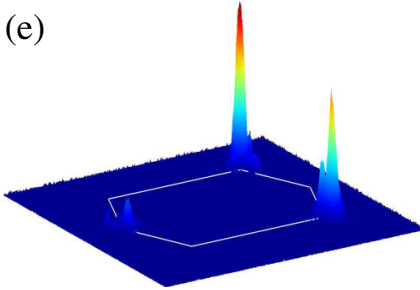

(f)

(d)
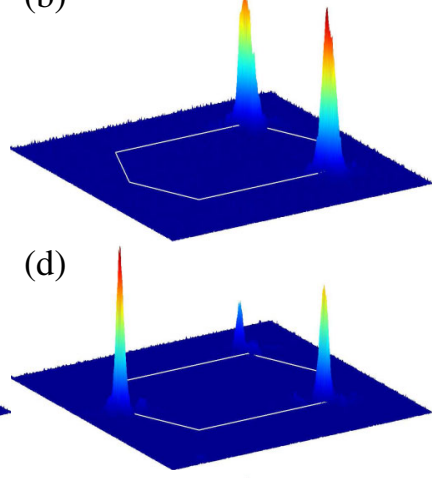

(g) Band populations, $\mathrm{M}$ - points

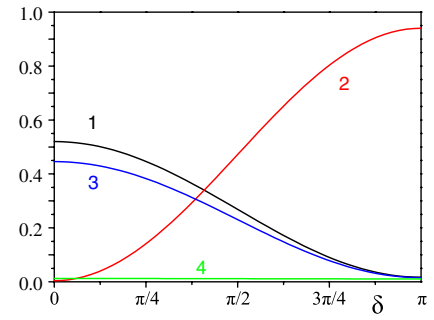

(h) Output relative powers

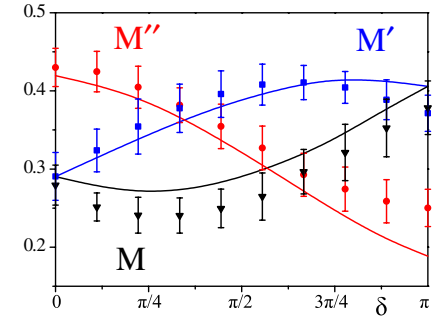

FIG. 3 (color online). Switching of power between different outputs by input phase-only manipulation. (a) Three-level Pendellösung oscillations realized by coupling of three $M$ points. The experimental input in (b) consists of two beams at $M$ and $M$ ' points with equal power and phase difference $\delta$. Corresponding LZM solution is derived in [20], and it allows one to obtain a single beam at the output, as in (c) with $\delta=0$, or any pair of output beams in (d)-(f) with $\delta=\pi / 3$ in (d), $\pi$ in (e), and $5 \pi / 3$ in (f). The Bloch-band populations in (g) (calculated for $I_{0}=1.1$ and $E_{\text {ext }}=1 \mathrm{kV} / \mathrm{cm}$ ) show the coupling between first three bands, as expected from resonant theory. (h) Measured relative powers of the output beams (symbols and error bars) and the corresponding numerical simulations (solid lines). $E_{\mathrm{ext}}=1 \mathrm{kV} / \mathrm{cm}$.

of populations between high-symmetry points in momentum space, while the Bloch-band populations in Figs. 2(d) and $3(\mathrm{~g})$ are given by the initial excitation. To induce tunneling between different Bloch bands, one needs to break the periodicity of the lattice by additional longitudinal modulation $[23,24]$ or a linear refractive index gradient $[7,8]$. The latter case corresponds to LZ tunneling and has been previously studied in square lattices [8]. However, no relation between Pendellösung oscillations and the tunneling process has been revealed. The reduction from Eq. (1) to Eq. (2) suggests that, for small propagation distances, the Pendellösung oscillations will dominate the dynamics, while the tunneling process determines the asymptotic power transport. 

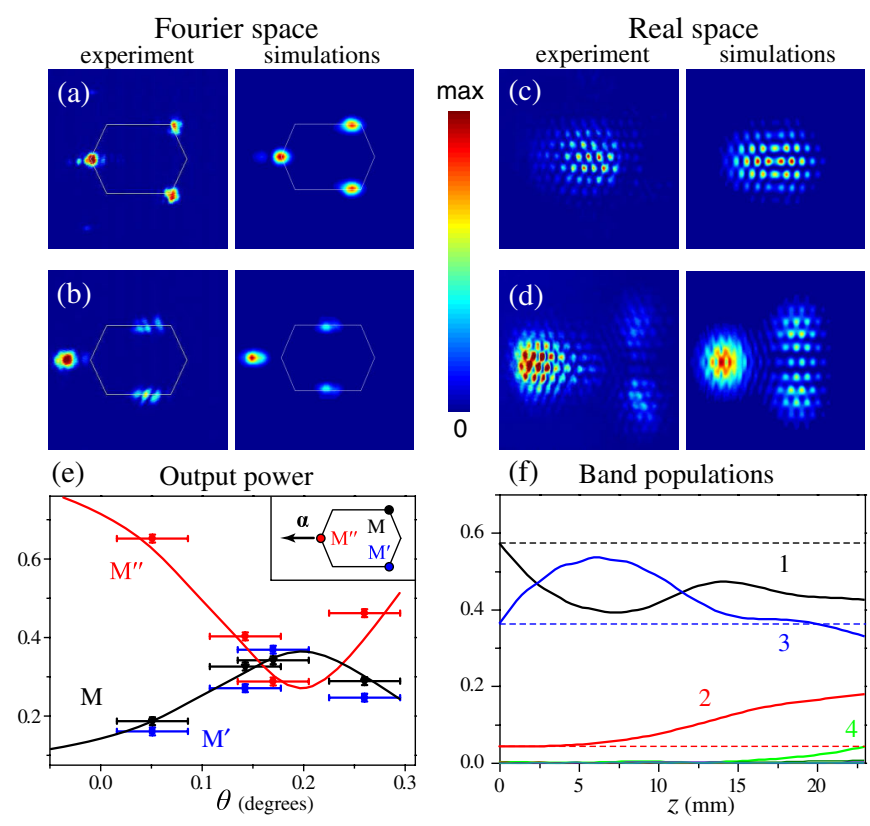

(f) Band populations

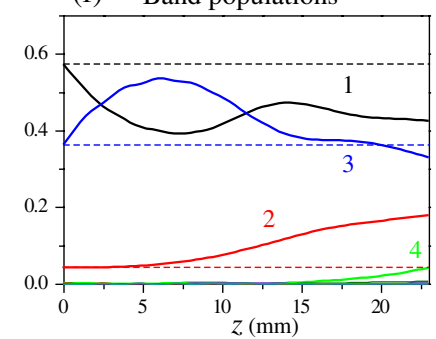

FIG. 4 (color online). Observation of Landau-Zener tunneling. (a),(b) Fourier space and (c),(d) real space output intensity distributions for input inclination angles of the signal beam: $\theta=0.14^{\circ}$ in (a),(c) and $\theta=0.26^{\circ}$ in (b),(d). (e) Measured output power ratios (markers) and the corresponding numerical simulations (solid lines). The error bars show finite beam widths in the reciprocal space; the arrow in the BZ inset indicates the direction of the linear index gradient $\alpha=\nabla I_{\text {grad }}$. (f) The dynamics of band populations (in the Bloch-wave basis of the periodic lattice with $\alpha=0$ ) during the tunneling in the crystal with band indices next to the curves. Calculations are for $I_{0}=0.9$ and $E_{\text {ext }}=1.35 \mathrm{kV} / \mathrm{cm}$, with the gradient illumination $I_{\text {grad }}$ of width $b=200 \mu \mathrm{m}$ and amplitude $B=2$ [20].

In order to study experimentally the tunneling dynamics of optical beams in lattices with index gradient, the crystal is illuminated from the top with a transversely modulated incoherent white light which induces a refractive index gradient along the transverse $x$ direction. Since it is not possible to directly observe the evolution of the signal beam inside the crystal, we vary the incident angle and image the output real space and the Fourier space onto a CCD camera [8]. For a fixed crystal length and angles below the Bragg resonance, such an excitation at different transverse wave vector components is equivalent to different starting points in the BZ and thus allows one to infer details of the tunneling dynamics at different stages of the beam evolution. Our results for symmetric LZ tunneling in Fig. 4 confirm theoretical predictions and demonstrate the interplay of both effects at the initial stage of evolution. Since the medium length $L$ is relatively short, it is not possible to reach the asymptotic transfer of population between bands, yet we observe significant transfer of power between resonantly coupled wave packets in Fourier space, shown in Fig. 4(e). The localization of these waves in momentum space corresponds to high-symmetry points in the frame moving in the BZ due to gradientinduced Bloch oscillations, cf. Figs. 4(a) and 4(b). The actual interband coupling is demonstrated in numerical simulations in Fig. 4(f), where the first and third Bloch bands undergo periodic exchange of energy with small and gradual tunneling of power to the second band. Therefore, such regime can be characterized as quasi-Pendellösung oscillations.

In conclusion, we have studied Pendellösung oscillations and interband Landau-Zener transitions in experiments on resonant coupling between high-symmetry points of 2D photonic lattices. The comparison of both effects shows that, in biased lattices of a finite length, the Landau-Zener tunneling is dominated by Pendellösung oscillations allowing for spatial spectral shaping of the waves. Our findings provide an important insight into resonant wave transport in periodic media, and they can be applied to electromagnetic and matter waves.

This work was supported by the Australian Research Council and the German Academic Exchange Service.

*Present address: Laboratory for Micro- and Nanotechnology, Paul Scherrer Institut, 5232 Villigen, Switzerland.

[1] C. G. Shull, Phys. Rev. Lett. 21, 1585 (1968).

[2] P. J. Martin, Phys. Rev. Lett. 60, 515 (1988).

[3] J. D. Joannopoulos et al., Photonic Crystals: Molding the Flow of Light (Princeton, Princeton, NJ, 2008), 2nd ed.

[4] M. L. Calvo et al., Phys. Rev. Lett. 97, 084801 (2006).

[5] S. Savo, Opt. Express 16, 9097 (2008).

[6] V.S. Shchesnovich and S. Chavez-Cerda, Opt. Lett. 32, 1920 (2007); V.S. Shchesnovich, A. S. Desyatnikov, and Yu. S. Kivshar, Opt. Express 16, 14076 (2008).

[7] H. Trompeter et al., Phys. Rev. Lett. 96, 023901 (2006).

[8] H. Trompeter et al., Phys. Rev. Lett. 96, 053903 (2006).

[9] A. Yariv, Quantum Electronics (J. Wiley \& Sons, New York, 1989).

[10] F. Lederer et al., Phys. Rep. 463, 1 (2008).

[11] N. K. Efremidis et al., Phys. Rev. E 66, 046602 (2002).

[12] S. Longhi, Laser Photon. Rev. 3, 243 (2009).

[13] I. Bloch, Nature Phys. 1, 23 (2005); O. Morsch and M. Oberthaler, Rev. Mod. Phys. 78, 179 (2006).

[14] U. Peschel, T. Pertsch, and F. Lederer, Opt. Lett. 23, 1701 (1998).

[15] J. W. Fleischer et al., Nature (London) 422, 147 (2003).

[16] T. Schwartz et al., Nature (London) 446, 52 (2007).

[17] A. Szameit et al., Nature Phys. 5, 271 (2009).

[18] G. Bartal et al., Phys. Rev. Lett. 95, 053904 (2005).

[19] V. S. Shchesnovich et al., Phys. Rev. E 74, 056602 (2006); A.S. Desyatnikov et al., Opt. Lett. 32, 325 (2007).

[20] See supplemental material at http://link.aps.org/ supplemental/10.1103/PhysRevLett.106.083902 for a theoretical model.

[21] M. Grifoni and P. Hänggi, Phys. Rep. 304, 229 (1998).

[22] B. Terhalle et al., Phys. Rev. Lett. 101, 013903 (2008); Opt. Lett. 35, 604 (2010).

[23] Y. V. Kartashov, V. A. Vysloukh, and L. Torner, Phys. Rev. Lett. 99, 233903 (2007).

[24] K. G. Makris et al., Opt. Express 16, 10309 (2008); K. Shandarova et al., Phys. Rev. Lett. 102, 123905 (2009). 